\title{
Design of Low Phase Noise and Wide Tuning Range Voltage Controlled Oscillator for Modern Communication System
}

\author{
Rachita Singh*, Rajat Dixit** \\ *(Department of Electronics and Communication Engineering, Jaypee Institute of Information Technology, \\ Noida) \\ ** (Department of Electronics and Communication Engineering, Jaypee Institute of Information Technology, \\ Noida)
}

\begin{abstract}
Voltage Controlled Oscillator is one of the most imperative blocks in the present communication system. It finds application in both wired as well as wireless communication as clock generator, frequency synthesizer and system synchronizer. The desirable characteristics of a VCO are high frequency, low phase noise, low power, low voltage and enhanced range of tuning. A three stage CMOS voltage controlled ring oscillator based on differential architecture and a LC tank voltage-controlled ring oscillator based on cross coupled architecture hasbeen designed by using 32nm and $90 \mathrm{~nm}$ technology respectively. The former VCO has a tuning range spanning from $733.718 \mathrm{MHz}$ to $2.065 \mathrm{GHz}$ with a phase noise of $-79.4 \mathrm{dBc} / \mathrm{Hz}$ while the latter possess better phase noise of $-89.7 \mathrm{dBc} / \mathrm{Hz}$ at the cost of reduced tuning range spanning from $4.5 \mathrm{GHZ}$ to $4.9 \mathrm{GHZ}$ only which is comparatively lesser than its counterpart.
\end{abstract}

Keywords: Differential ended Ring Oscillator, LC Tank Oscillator, Phase Noise, Tuning Range, Voltage Controlled Oscillator

\section{INTRODUCTION}

Oscillators are a central part in numerous electronic frameworks. Applications utilizing oscillators encompass clock generation in microchips to frequency translation in cell phones. Distinctive application likewise requires diverse arrangement of oscillator execution parameters. As today's integrated circuits are converging towards CMOS, the plan of robust and high performance CMOS oscillators, all the more particularly, voltage-controlled oscillators (VCOs), has turned out to be extremely important.

The key measurements of a VCO comprise of: frequency of oscillation, tuning range, phase noise, and power utilization. The oscillation frequency is controlled by the application in which the VCO is utilized as a part of, for example, microprocessor or mobile phone. The tuning range is ascertained by the need of the application and the oscillation frequency variation on the account of process and temperature changes. The center frequency of some CMOS oscillators may differ by a variable of two at the extremes of process and temperature [1], therefore a wide tuning range is exceptionally attractive. Low phase noise VCOs has turned into a subject of research owing to immense development in wireless communication. Accordingly the necessity of phase noise in the oscillator turns out to be more stringent. In advanced microprocessors, the phase noise of the oscillator will influence the jitter of the clock signal and the timing margin, hence confines framework execution. In conclusion, power utilization is critical for mobile applications, for example, phones and portable PCs where a battery supply the power. A low power configuration will expand the battery life and provide long term power autonomy.

\subsection{Ring VCO}

\section{VCO TOPOLOGIES}

A ring oscillator comprises of number of delay stages in a loop, which forms an unstable negative feedback circuit [2-6]. Fundamental ring oscillator utilizes single-ended CMOS inverters instead of amplification stages as required in LC oscillators.No less than three inverters are required to form voltage-feedback loop to start oscillation.From the review of this circuit, it is observed that circuit has no stable operating point. The DC operating point at logic threshold voltage,input and output voltages are equivalent for all inverters. $V_{\text {th }}$ is characteristically unstable in the sense that any disturbance in node voltage would make the circuit to drift away from the DC operating point. Hence, a closed loop cascade connection of any odd number of inverters will show astable conduct i.e. such a circuit will oscillate once anyone of the inverter will deviate off from the unstable operating point, $\mathrm{V}_{\text {th }}$. Subsequently, the circuit is called Ring Oscillator.There are mainly two models accessible which can be chosen depending upon the application and complexity of ring oscillator. The single-ended signal ring VCO is easy to implement, 
yet when it is incorporated with other applications, the VCO output is influenced by other circuits. Most of the systems utilize differential pair VCO topology.

In single-ended ring oscillators, majority of the devices encounters complete switching amongst on and off states. The large signal swing and sharp transitions improve the noise performance. Inspite of such merits, single-ended ring oscillators experience the ill effects of substrate and supply noise. Hence differential pair ring oscillator was utilized due to the accompanying reasons: immunity to common mode noise, $50 \%$ duty cycle at output, even number of stages if output feedback is interchanged and improved spectral purity.

\subsection{LC Tank VCO}

The LC-Tank is constructed by an inductor and a capacitor shown as Fig.1 so the resonant frequency is $\omega=1 / \sqrt{L C}$, which means at the frequency $\omega$ the impedance of the LC-Tank is infinite (the impedance of the inductor is $j \omega L$ and the capacitor is $1 / j \omega C$ ). If some energy is stored in the tank, it will generate a periodic signal with frequency $\omega$ which is the oscillator. The factor $Q$ can be defined as[12]:

$$
Q=2 \pi . \frac{\text { Energy stored }}{\text { energy dissipated per cycle }}
$$

This, however, is an ideal circuit; in reality, the inductor and wires all have the parasitic resistors.

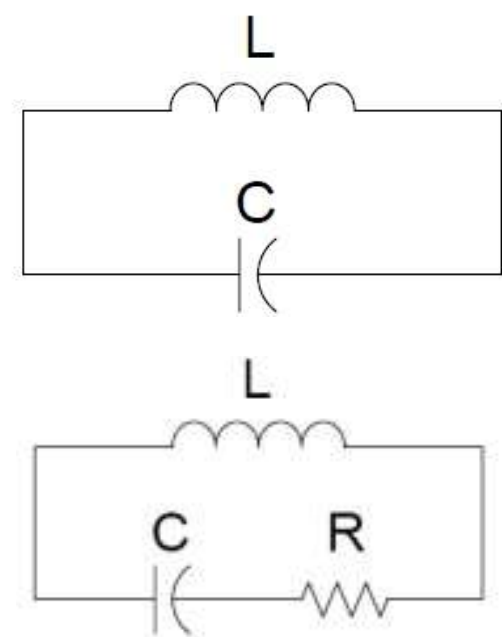

Fig.1(a) Ideal LC Tank (b) Non-Ideal LC Tank For non-ideal LC-Tank, the impedance can be formulated as:

$$
Z=\frac{s(R+L)}{1+R C s+L C s^{2}}
$$

From Fig.1 the non-ideal LC-Tank is unable to provide stable periodic signal at frequency $\omega$, because the energy in the tank will be devoured by the resistor. Therefore, active circuit must be included in the non-ideal LC-Tank to adjust for the resistive effects.
For the most part, the noise attributes of the LC resonant VCO is superior to its ring counterpart, since the inductor and capacitor possess high quality factor. The resonant tank can efficiently utilize energy for oscillation.For few applications, particularly in communication frameworks, for example, cell phones or wireless sensor, the communication speed and bit error rate (BER) must be kept up. In this manner, they require the $\mathrm{LC} \mathrm{VCO}$, in light of the fact that it can give better phase noise. On the other hand, inductor and varactors consumes considerably larger area in contrast with the standard CMOS transistors, the LC VCO must be utilized on certain high cost system. Additionally, with the variety of communication standards including wired and wireless existing in a system, wide frequency band VCOs are required [912]. The LC VCO can't deliver wide tunability since the inductors and varactors are not effortlessly tuned. Thus, ring designs and circuit strategies are examined to accomplish better frequency tuning band and comparable phase noise characteristics.

\section{DESIGN IMPLEMENTATION}

\subsection{Differential ended Ring Oscillator}

A control voltage isfed into the VI convertor, thus generating a control current. This control current tunes the current controlled oscillator (CCO) to generate differential clock signals. Finally the buffer stage converts the differential signals to single-ended and square wave clock signal is generated.The proposed model for ring oscillator is clearly depicted in Fig.2.

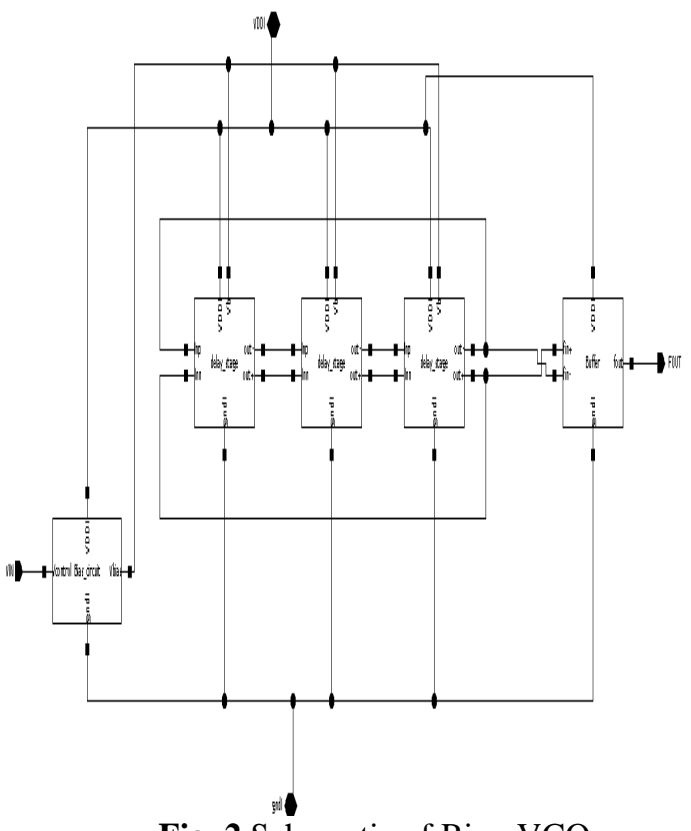

Fig. 2 Schematic of Ring VCO 


\subsubsection{Bias Circuit}

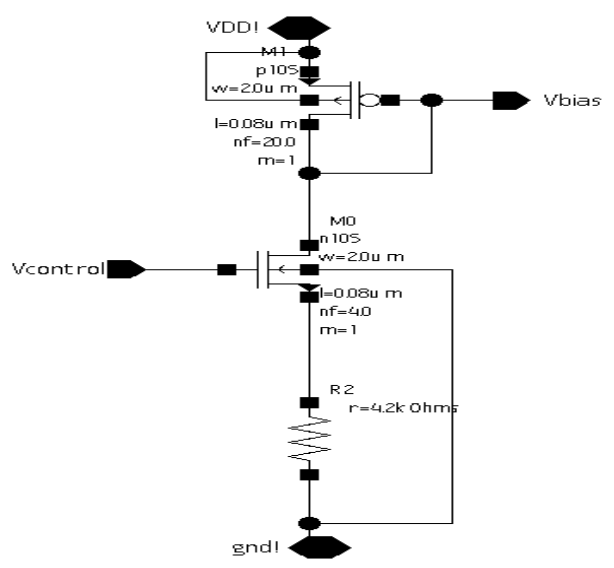

Fig. 3 Bias Circuitry

In order to get linear voltage to current conversion, the aspect ratio of M1 transistor must be large, for a small $V_{\text {overdrive }}$.A first order relationship between $V_{\text {control }}$ and $I_{\text {control }}$ has been derived below. $V_{G S}$ of $M_{1}$ :

$V_{\text {control }}-I_{\text {control }} \times R=V_{t h}+V_{o v}=V_{t h}+\sqrt{\frac{2 I_{\text {control }}}{K^{\prime}(W / L)}}(3)$

If $\frac{W}{L}$ of $M_{1}$ is very large then:

$$
V_{\text {control }} \cong V_{\text {th }}+I_{\text {control }} \times R
$$

\subsubsection{Delay Cell}

By varying $I_{\text {control }}$ a current-controlled CSA-based ring oscillator is formed with an output voltage swing.

$$
\begin{gathered}
\Delta v=V_{O H}-V_{O L}=V_{T H}+\sqrt{\frac{(W / L)_{1}-(W / L)_{2}}{(W / L)_{1} \cdot\left(W / L I_{2}\right.} \frac{2 \text { control }}{K^{\prime}}}(5) \\
f_{\text {osc }} \propto \frac{I_{\text {control }}}{N C_{L} \Delta V} \propto \sqrt{I_{\text {control }}}
\end{gathered}
$$

$\mathrm{N}$ : No. of delay stages in the ring oscillator.

$C_{L}$ : Output load capacitance.

For a fixed $I_{\text {control }}$ range, this relationship can be approximated as Quasi-linear relationship.The advantage of the current controlled CSA delay stage is that the ground noise coupled from other circuitry within the chip is rejected by the CSA as a common mode noise because both the input and output are reformed to the same ground. This concept of currentcontrolled CSA delay cell can be used by the differential topology.

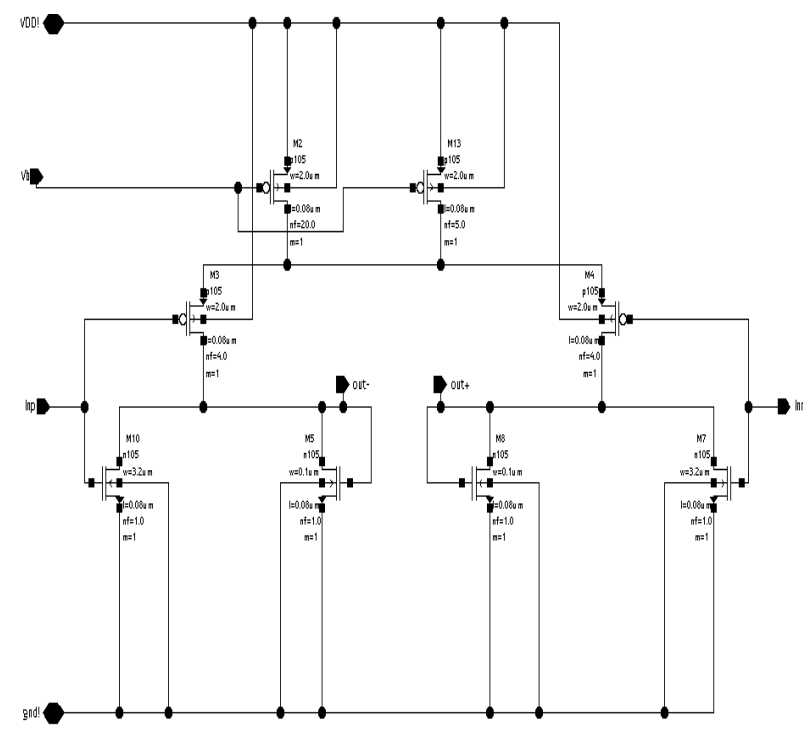

Fig. 4Delay Cell

\subsubsection{Buffer Circuit}

The waveform of VCO output is nearly sinusoidal waveform with a limited voltage swing. So it must be shaped before applying it to digital circuit.In order to convert VCO output signal to square rail-to-rail switching signal, a buffer stage is added at the end of the VCO delay cell.

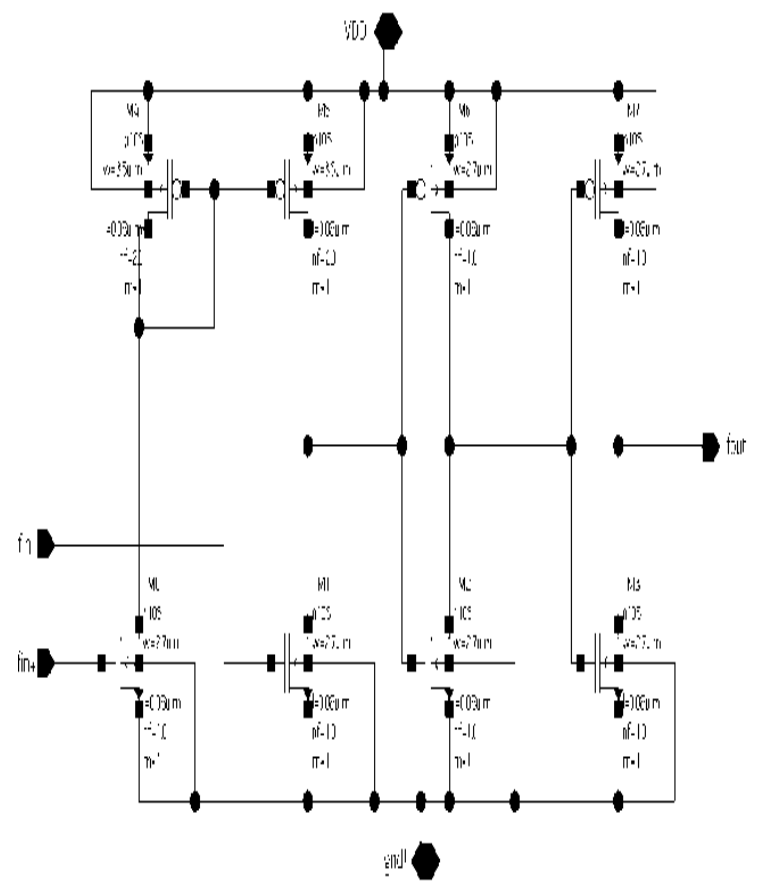

Fig. 5Buffer Circuit 


\subsection{LC Tank VCO}

The proposed model for LC Tank VCO is depicted in Fig.6.

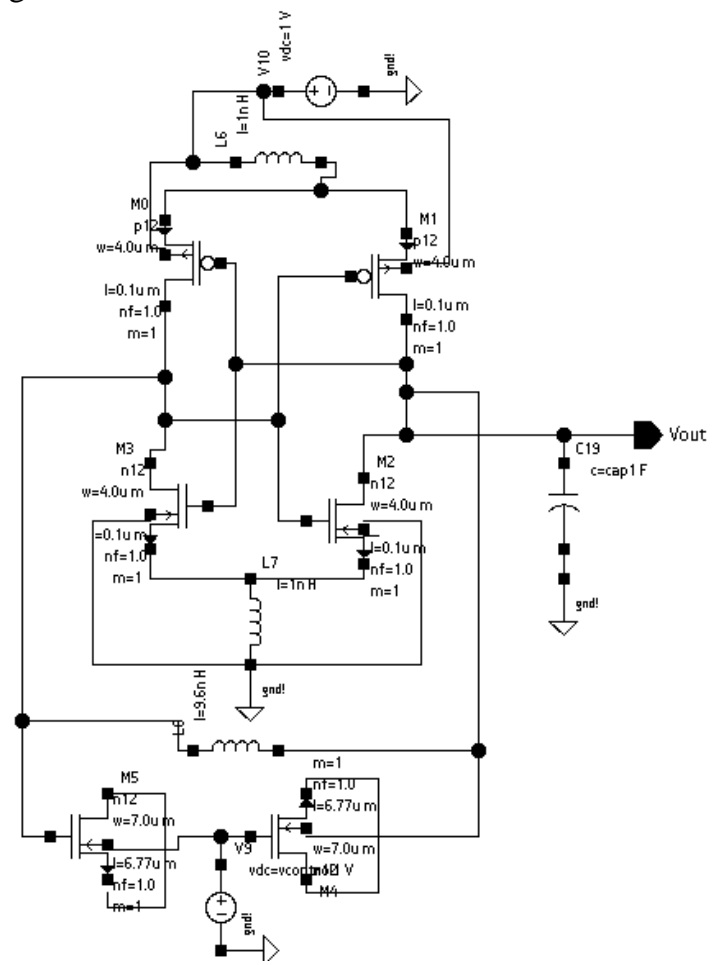

Fig. 6LC Tank VCO

\section{SIMULATION RESULTS}

4.1 Simulation Results of Differential ended Ring Oscillator

4.1.1 Transient Characteristics

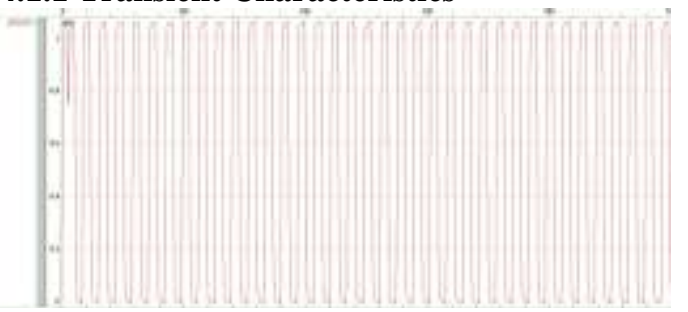

Fig. 7Transient Characteristics of Ring VCO

Here Fig.7 demonstrates the oscillatory output of the differential ended ring VCO. Its voltage swings from $0 \mathrm{~V}$ to $1.0 \mathrm{~V}$ that is equivalent to the biased potential. The shape of the output voltage swing is square due to buffer stage.

\subsubsection{Tuning Characteristics}

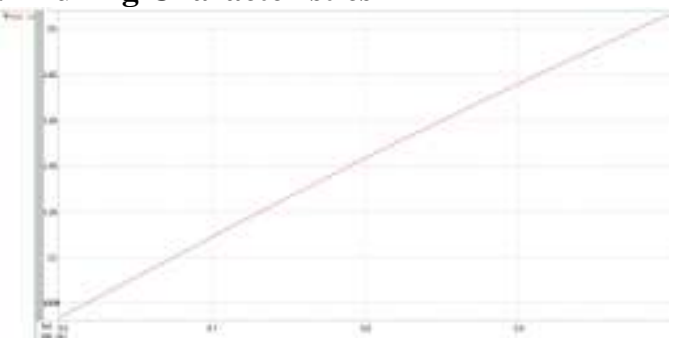

Fig. 8Tuning Characteristics of Ring VCO

In VCO the output frequency is a function of control voltage. That is the output frequency linearly varies with the control voltage. Fig. 8 shows the linear variation of frequency with respect to control voltage. Here as voltage is changing from $0.6 \mathrm{~V}$ to $1.0 \mathrm{~V}$ frequency range is changing from $790 \mathrm{MHz}$ to $2 \mathrm{GHz}$.

\subsubsection{Phase Noise Variation}

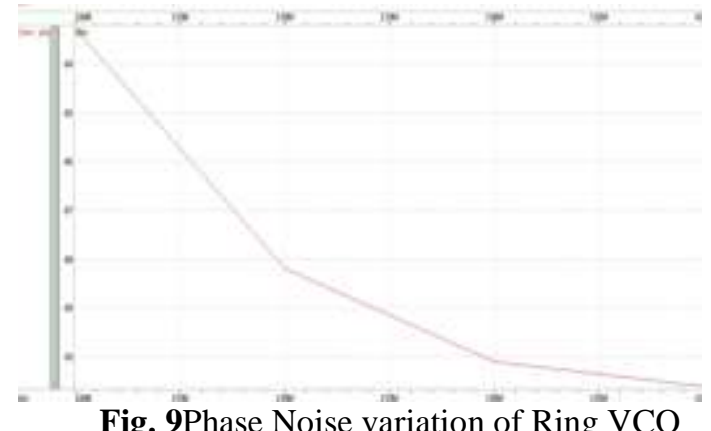

Fig.9 shows variation of phase noise with respect to operating frequency of differential-ended ring VCO. Since $1 / \mathrm{f}$ noise is predominant at lower frequency, it also affects phase noise. The rate of degradation in phase noise will be higher at lower frequency and converse at higher frequency as the effect of flicker noise decreases and thermal noise become dominant. The phase noise for this model is $79.4 \mathrm{~dB} / \mathrm{Hz}$.

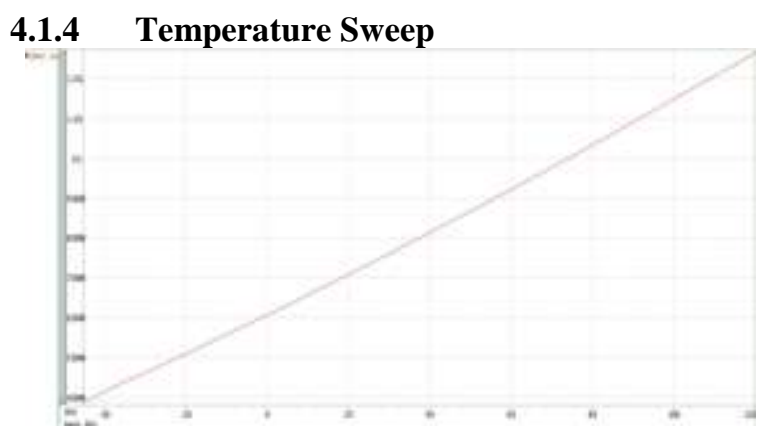

Fig. 10Temperature Sweep of Ring VCO

Fig.10 shows dependence of oscillatory frequency on the temperature where frequency varies linearly with the variation of temperature from -40 to 125.

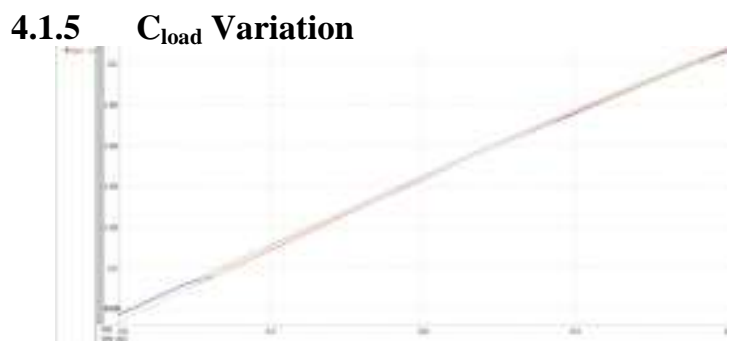

Fig. $\mathbf{1 1 C}_{\text {load }}$ Variation of Ring VCO 
Fig.11 shows the effect on tuning frequency with respect to control voltage as the output capacitive load varies from $1 \mathrm{fF}$ to $120 \mathrm{fF}$.

\subsection{Simulation results of LC Tank oscillator}

\subsubsection{Transient Characteristics}

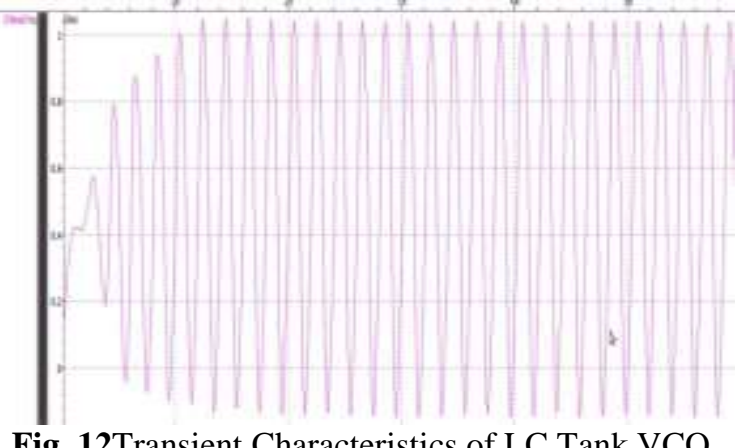

Fig. 12Transient Characteristics of LC Tank VCO

Fig.12 represents the transient characteristic of LC tank VCO. The output waveform is sinusoidal as no buffer stage has been applied and acceptable voltage swing is from $0 \mathrm{~V}$ to $1 \mathrm{~V}$.

\subsubsection{Tuning Characteristics}

Fig.13 represents the tuning characteristics of LC tank VCO. The tuning frequency is the function of control voltage. LC tank circuit does not supports linear characteristic for the control voltage lower than the $0.7 \mathrm{~V}$.

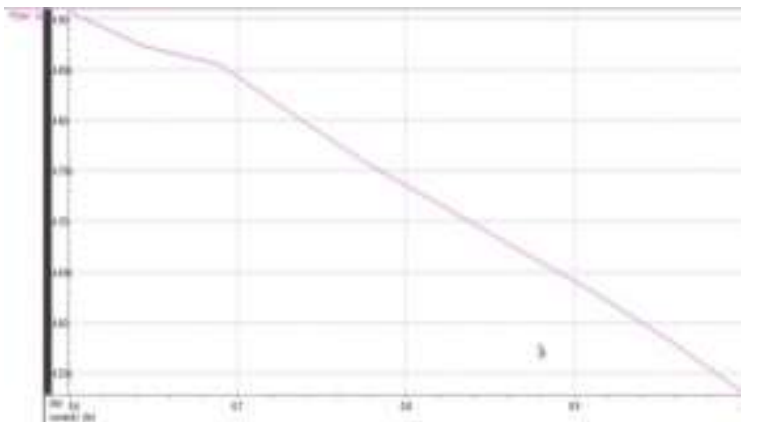

Fig. 13Tuning Characteristics of LC Tank VCO

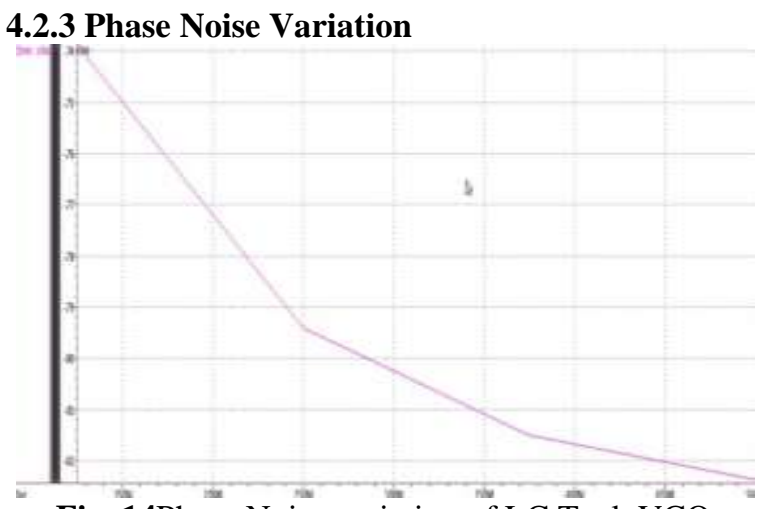

Fig. 14Phase Noise variation of LC Tank VCO
The concept of phase noise variation is similar to differential-ended ring VCO. But it possessesbetter noise performance of $-89.7 \mathrm{dBc} / \mathrm{Hz}$. Table 1 depicts the specification comparison of both the topologies.

Table 1Specification Comparison of Ring VCO and LC Tank VCO

\begin{tabular}{|c|c|c|}
\hline \multicolumn{3}{|c|}{ Specifications companisen } \\
\hline Parameters & $\begin{array}{c}\text { Differential-ended } \\
\text { ring VCO }\end{array}$ & LC-tank VCO \\
\hline $\begin{array}{c}\text { Minimum input } \\
\text { voltage }\end{array}$ & $0.6 \mathrm{~V}$ & $0.6 \mathrm{~V}$ \\
\hline $\begin{array}{c}\text { Maximum Input } \\
\text { voltage }\end{array}$ & $1.0 \mathrm{~V}$ & $1.0 \mathrm{~V}$ \\
\hline Power supply & $1.0 \mathrm{~V}$ & $1.0 \mathrm{~V}$ \\
\hline $\begin{array}{c}\text { Minimum } \\
\text { frequency }\end{array}$ & $790 \mathrm{MHz}$ & $5.5 \mathrm{GHz}$ \\
\hline $\begin{array}{c}\text { Maximum } \\
\text { frequency }\end{array}$ & $2.009 \mathrm{GHz}$ & $5.9 \mathrm{GHz}$ \\
\hline Tuming range & $1200 \mathrm{MHz}$ & $400 \mathrm{MHZ}$ \\
\hline Phase Noise & $-79.4 \mathrm{dBc} / \mathrm{HZ}$ & $-89.7 \mathrm{dBc} / \mathrm{Hz}$ \\
\hline $\begin{array}{c}\text { Temperature } \\
\text { sustainable range }\end{array}$ & $-40^{\circ} \mathrm{C}$ to $125^{\circ} \mathrm{C}$ & $-40^{\circ} \mathrm{C}$ to $125^{\circ} \mathrm{C}$ \\
\hline $\begin{array}{c}\text { Output capacitor } \\
\text { max load }\end{array}$ & $20 \mathrm{~F}$ & $35 \mathrm{~F}$ \\
\hline
\end{tabular}

\section{CONCLUSION}

In this paper, the design and analysis of a wide tuning range, low phase noise CMOS voltagecontrolled ring oscillator has been presented. A differential-pair voltage controlled ring oscillators (VCRO) was realized because of its inherent advantages over the single-ended structures. The proposed designemploys the basic concepts VCO which comprises of a voltage to current convertor ( $\mathrm{V}$ I) and current controlled oscillator (CCO).The delay cell comprised ofdifferential current controlled current steering amplifier (CSA). An additional buffer stage is used in order to get the rail-to-rail swing at the output.Further a LC-Tank VCO is simulated and is compared with the differential-pair voltage controlled ring oscillators. The LC-Tank possess better phase noise performance while differential-pair voltage controlled ring oscillators possess wider tuning range as compared to their counterpart. As a result, Phase locked loop requiring wide tuning range generally use ring VCO and inductor and capacitor (LC) tank VCO topology is used for frequency translation in mobile phones for its relatively high oscillation frequency and low phase noise.

\section{REFERENCES}

[1] J. N. Burghartz, Silicon RF Technology The Two Generic Approaches, Proc.ESSDERC, pp.143-153, 1998.

[2] Abidi, Phase Noise and Jitter in CMOS Ring Oscillators, IEEE J Solid-State Circuits, Vol. 41, No. 8, Aug 2006.

[3] Y. Kao and M.Hsu, Theoretical Analysis of Low Phase Noise Design of CMOS 
VCO,IEEE Microwave and Wireless Components Letters, Vol.15, No.1, Jan2005.

[4] P. Larsson, Measurement and Analysis of PLL jitter Caused by Digital Switching Noise, IEEE J. Solid State Circuits, Vol. 36, No.7, Jul 2001.

[5] B. Razavi, A 2-GHz 1.6-mW Phase-Locked Loop, IEEE J. Solid-State Circuits, Vol. 32, No. 5, May 1997.

[6] T. Nian et al., A 1-V CMOS VCO for 60$\mathrm{GHz}$ Applications, Proc. IEEE Asia-Pacific Microwave Conference (APMC), 2005.

[7] P. Larsson, A2-1600-MHz CMOS Clock Recovery PLL with Low $\mathrm{V}_{\mathrm{dd}}$ Capability, IEEE J. Solid-State Circuit, Vol. 34, No.12, Dec 1999.

[8] B. Razavi and J.J. Sung, A $6 \mathrm{GHz} 60 \mathrm{~mW}$ BiCMOS Phase-Locked Loop, IEEE JSolidState Circuits,Vo1.29, no.12, pp.1560-1565, Dec 1994.

[9] S.K. Enam and A. Abidi, A Gigahertz Voltage Controlled Ring Oscillator, Electron. Letters, Vol 22, pp.677-679, Jun 1986.

[10] Yalcin Alper Eken, A 5.9GHZ Voltage Controlled Ring Oscillator in 0.18um CMOS, IEEE Journal of Solid State Circuits, Vol.39; No.1, Jan 2004.

[11] William Shing Tak Yan and Howard cam Luwng, A 900MHZ CMOS Low Phase Noise Voltage Controlled Ring Oscillator, IEEE transactions on Circuit and System-ii, Analog and Digital Signal Processing, Vol. 48, No.2, Feb 2009.

[12] Hajimiri and T. H. Lee, Design issues in CMOS differential LC oscillators, IEEE Journal of Solid State Circuits, 34(5), May 1999. 